\title{
A blank page: feedback from first referral hospitals to primary health care clinics
}

\author{
TL Legodia, $\mathrm{i}^{(}$and JE Wolvaardt ${ }^{\mathrm{a} *}$ \\ aschool of Health Systems and Public Health, University of Pretoria, Pretoria, South Africa \\ *Corresponding author, email: liz.wolvaardt@up.ac.za
}

Background: Primary care practitioners depend on the feedback from hospitals in order to care for returning patients effectively. Lack of such feedback from the hospitals leads to frustration, poor efficiency and care that it is not cost effective. This study examined the presence and adequacy of written feedback from first referral hospitals to primary health clinics (PHC) in the Metsweding district, Tshwane, South Africa.

Methods: A retrospective descriptive stratified cluster study was done by reviewing 863 patient records selected randomly from referred patients between 1 January 2010 and 31 December 2011 in 6 of the 11 clinics. The clinics were stratified according to type of setting and 6 clinics sampled proportionately.

Results: Of the 858 referrals only 5.4\% $(n=46)$ had feedback letters. Details of the patient were listed in $35(76.0 \%)$ of the feedback letters. There were no contact details for the referring institution or practitioner in almost half of the feedback letters (41.8\%; $n=19)$. Most did not mention the symptoms $(87 \% ; n=40)$ or signs $(89.2 \% ; n=41)$ but the majority $(58.6 \% ; n=27)$ recorded the condition or diagnosis of the patient. The follow-up instructions were documented in 30 letters (65.2\%).

Conclusions: The low rate of return feedback letters appears to be a commonplace experience and this study both quantifies the extent of this problem and highlights the weaknesses in the letters themselves. The findings reinforce the experiences of practitioners in PHCs and have practical implications for hospital doctors and district hospital managers who wish to strengthen the health system.

Keywords: communication, feedback, primary health care, referral

\section{Introduction}

An efficient referral system is an important part of a well-functioning health care system at the local, provincial and national level of a country.

Clinics are expected to refer patients to referral hospitals for care that cannot be given at a primary level of care. The World Health Organization (WHO) Expert Committee on the Role of Hospitals highlights the full involvement of primary health care (PHC) clinics as the first point in a referral system. ${ }^{1}$ The role of hospitals is clarified as the component of the district health system that immediately supports the health activities of the district or community and especially supports the primary health care activities. ${ }^{1}$ A health system based on primary health care cannot be realised without the support of a network of hospitals; it requires the back up of hospitals in promotive and preventative as well as curative and rehabilitative services. ${ }^{2}$ The role of hospitals is defined as one that treats patients as best it can, discharges patients as soon as it is consistent with appropriate care and explains to the referring practitioner or health care worker the clinical findings and further care required. ${ }^{2}$ Some studies have shown that there is poor use of information as well as a poor standard of both referral and feedback letters. $^{3,4}$

Opportunities for learning may be missed or reduced through the poor communication between the specialists (or hospital-based doctors) and the primary health care practitioners, ${ }^{5}$ and continuity of care may be negatively affected and contribute to adverse events. ${ }^{6}$ Several academic specialists found patient-specific feedback to be cost effective. ${ }^{7}$ Lack of adequate feedback from hospitals was believed to be one of the reasons for low referral rates from clinics to hospitals and is a reflection of frustration by the primary health care practitioners. ${ }^{8}$ Siddiqi, Kielmann and Khan reported that there was no feedback at all from the hospitals to the primary level of care in their study in Pakistan. ${ }^{9}$ A similar study conducted in the Republic of Honduras reported that there was only a $1.4 \%$ response rate to referrals. ${ }^{10}$ Despite lack of agreement on the desired features of feedback letters or replies, ${ }^{11,12,13}$ it is suggested that consensus on what features should be included should be reached between the specialists (or hospital-based doctors) and the general practitioners (GPs) at the primary care level. ${ }^{14}$ Items viewed by both the specialists and the general practitioners as always important to include in the feedback letters were: mention of the patient's problem (symptoms, signs or condition), the clinical management plan, the treating doctor's signature or name, findings on investigation, the follow-up appointment of the patient and a summary of the presenting clinical history of the patient. ${ }^{15}$

The South African public health system is characterised by a hierarchical structure and referral system. The role of district hospitals is to provide level one (generalist) services to in- and outpatients referred from PHC clinics and community health centres. ${ }^{16} \mathrm{PHC}$ clinics are generally run by nurses and belong either to one of the nine provincial departments of health or to local authorities/municipalities.

The aim of the study was to assess the feedback received from three referral hospitals to the local authority PHC clinics in the Metsweding district with the aim of determining whether there is adequate feedback to these clinics as part of an effective referral system. In particular the study explored the presence or absence of feedback and the adequacy of the content in the feedback letter. 
Table 1: The sample size and type of setting of each clinic in the sample

\begin{tabular}{lcccccc}
\hline Clinic & A & B & C & D & E & F \\
\hline Setting & S-u & Ru & Ur & Ru & S-u & S-u \\
No. of files & 126 & 70 & 88 & 67 & 92 & 420 \\
\% of total & 15 & 8 & 10 & 7 & 11 & 49 \\
\hline
\end{tabular}

Notes: S-u: Semi-urban; Ru: Rural; Ur: Urban.

\section{Research methods and design}

\section{Study design}

A descriptive retrospective stratified cluster study design was used.

\section{Setting}

The Metsweding district municipality (MDM) is situated in the north-eastern part of Gauteng province, South Africa. Metsweding has since been disestablished and absorbed into the Tshwane Metropolitan Municipality. MDM consisted of two local municipalities, namely Kungwini and Nokeng-tsa-Taemane. ${ }^{17}$ The area is mostly rural and semi-urban and has substantial infrastructural problems: rural roads, and poor sanitation, water supply and transportation. ${ }^{17}$ The population of Metsweding is mainly uneducated, unemployed and poor. ${ }^{17}$

\section{Study population and sampling strategy}

The records of 863 adult patients referred from the local authority PHC clinics in Metsweding to the various clinical departments at the three referral hospitals (one in Metsweding, one in Gauteng and one public-private hospital) from 1 January 2010 to 31 December 2011 were reviewed.

The records were randomly selected from 6 clinics out of the 11 clinics in the Metsweding geographical area. The clinics were stratified according to urban $(n=2)$, semi-urban $(n=6)$ and rural $(n=3)$ settings. Six clinics were sampled proportionately from the three strata resulting in a choice of one urban, three semi-urban and two rural clinics by way of random selection. The sample size from each clinic was proportional to the size of the clinic (Table 1).

\section{Data collection}

The patient files were drawn by the clinic clerks and the first author. Each file that formed part of the sample $(n=863)$ was reviewed by the first author to ensure that the file met the inclusion criteria of an adult patient who was referred for care at a district-level hospital in Metsweding. Most referred patients were sent to hospital via ambulance. Patients who were referred for radiology and colposcopy and patients who sought hospital care via other routes were excluded from this study. Similarly patients who were referred directly to a tertiary hospital were also excluded from the study.

A self-designed data-collection sheet was developed based on the factors described in the literature. ${ }^{15}$ This data-collection sheet was used by the first author to record the presence of the feedback letter from the hospital in the patient records and the details contained in the feedback letter. The quality of the data contained in the feedback letter was assessed by the first author (a clinician) looking at the presence of the following elements that are agreed upon as important in the literature: patient identification, the person who wrote the feedback letter's name and signature, description of the investigations done on the patient, the diagnosis of the patient, any treatment procedures or clinical management and a description of the necessary follow-up care of the patient.

\section{Data analysis}

The forms with the captured data were entered onto an Excel (Microsoft, Redmond, WA, USA) spreadsheet. Data were checked for accuracy and cleaned. Data analysis was done with the use of Excel.

\section{Ethical considerations}

The approval to conduct this study was obtained from the University of Pretoria Research and Ethics Committee (S45/2012). Permission to access the records was requested and obtained from the Department of Health Research Committee (2012/42) in Tshwane and from the clinic managers.

\section{Bias}

For the purposes of research both patient names and file numbers were used so the possibility that the referral letter was filed in a duplicate file is limited. It is possible that some patients lost their feedback letters, relocated to another district or died. The final sample size is small and therefore results cannot be generalised.

\section{Results}

The sample size was 863 and five records were spoilt and therefore omitted from the study. Of the 858 referrals to hospitals only $5.4 \%$ $(n=46)$ patient files had a feedback letter. The clinics in Metsweding have standard referral letters (there are three different designs and two of the designs have a feedback/reply section for the referral institution) to the hospitals but the hospitals do not appear to have a standard feedback letter or form. Each department appears to use its own feedback letter or form and the use of an unstructured memo or short summarised letter was commonplace.

The adequacy of the feedback letter was assessed by looking at the key features of the feedback letter (Table 2).

A positive feature of the feedback letters was that the details of the patient were listed in 35 (76\%) of the letters. Twenty-eight $(60.8 \%)$ of the replies had a signature of the replying health care practitioner. Follow-up instructions were documented in the majority of feedback letters $(65.2 \% ; n=30)$ with the responsible institution for follow-up indicated $(63.0 \% ; n=29)$. In general treatment recommendations were included $(63.0 \% ; n=29)$.

Among the negative features of the feedback letters was that almost half of the letters lacked the contact details of the referring institution or practitioner $(41.4 \% ; n=19)$. Most of the feedback letters did not mention the symptoms $(87 \% ; n=40)$ or clinical signs $(89.2 \% ; n=41)$ of the patients that were referred. The investigations done at the hospital were mentioned in less than half of the feedback letters $(36.9 \% ; n=17)$. Less than a quarter $(21.7 \% ; n$ $=10$ ) of feedback letters contained a follow-up date or time frame.

\section{Discussion}

The poor feedback rate of 5.4\% $(n=46)$ for the referral letters from the first referral hospital to the PHC clinics is similar to the findings reported in a study in the Republic of Honduras, ${ }^{10}$ which found that very few $(1.4 \% ; n=15 / 1072)$ of the total referral cases sent from the institution to another institution ( 870 hospitals, and 201 health cen- 
Table 2: Adequacy of the feedback letter $(n=46)$

\begin{tabular}{|c|c|c|}
\hline Variable & Feedback (\%) & (n) \\
\hline Details of patient stated on the feedback letter & 76.0 & 35 \\
\hline Follow-up instruction to clinic & 65.2 & 30 \\
\hline Responsible institution (clinic/hospital) for follow-up & 63.0 & 29 \\
\hline Treatment recommendations & 63.0 & 29 \\
\hline Feedback letter signed by replying health care practitioner & 60.8 & 28 \\
\hline Contact details of replying health care practitioner & 58.6 & 27 \\
\hline Reference to medication of patient & 58.6 & 27 \\
\hline Investigations done at the hospital mentioned & 36.9 & 17 \\
\hline Follow-up date/time frame indicated & 21.7 & 10 \\
\hline Symptoms of patient noted & 13.0 & 6 \\
\hline
\end{tabular}

tres) received a reply from the higher-level institutions (national hospitals, regional hospitals, area hospitals). In a study in Pakistan ${ }^{3}$ it was revealed that none of the higher-level facilities (hospitals) provided feedback to the first level of care facilities. One of the reasons for low referral rates from the clinics to the hospitals is suggested to be the lack of feedback. ${ }^{8}$ Clinics do not refer patients, as there is generally poor feedback and as a result referral is seen as a futile exercise.

Patient-specific feedback from hospitals is identified to be cost effective and related to improved outcome and reduced complications. ${ }^{7}$ The poor feedback in Metsweding could lead to low referral rates, be costly to both the patient and the health care department and lead to an overall poor outcome in the management of the patient.

In the study from the Republic of Honduras ${ }^{10}$ there was an observation that junior doctors complained of not receiving any feedback from the referral institutions, despite some of the referred patients presenting with complicated conditions. Considering the limited time of clinic hours and the budget constraints, simple diagnostic slips given to the patient could be sufficient. ${ }^{10}$

The feedback letters in this study contained inadequate information. The variance between the amount and quality of the information in the feedback letters could be explained by the use of an unstructured memo or short summarised letter that lacks the minimum necessary information needed by the PHC practitioners. Similar studies ${ }^{3,4,12}$ also reported a poor quality of referral letters and feedback reports. Even though 58.6\% $(n=27)$ of feedback letters in this study contained information on the condition (diagnosis) of the patient, very few $(13.0 \% ; n=6)$ mentioned the presenting symptoms of the patient and only five (10.8\%) contained information on the patient's clinical signs. These findings are poorer than those reported by van Walraven, Anthony and Weinberg. ${ }^{18}$ The authors examined the discharge summaries of urban city hospitals associated with the University of Ottawa and of the 106 charts with a discharge summary, information was missing on the admission diagnosis in 34\% $(n=36)$ of the summaries and the discharge diagnosis was missing in $25.5 \%(n=27)$. The conclusion was that there were considerable deficiencies in the completeness of the discharge summaries and the efficiency of the discharge summary system in the participating hospitals. ${ }^{18}$ The study by van Walraven, Anthony and Weinberg ${ }^{18}$ and this Metsweding study highlight the gaps in information concerning the overall presenting clinical picture of the patient and the condition of the patient at the hospital level. The deficiency in the information in the discharge summary or feedback letters reflects a missed opportunity for learning. Westerman, Hull, Bezenes and Gort maintain that opportunities for learning are reduced or missed through this poor communication between the hospital-based doctors and the primary health care general practitioners. ${ }^{5}$

The investigations done at the hospitals were only recorded in approximately a third of the feedback letters in this study $136.9 \%$; $n=17)$. This result is similar to the van Walvaren study where almost half $(42.9 \% ; n=115)$ of the 268 laboratory tests and results noted in the hospital charts were not reported in the discharge summary. ${ }^{18}$ Such a high percentage of missing recorded investigations in the discharge summaries or feedback letters implies that the clinic may have to repeat the investigations done at the hospital leading to duplication of work already done.

In Metsweding the follow-up date was commonly not included $(78.3 \% ; n=36)$. The follow-up clinical instructions to the clinic were stated in 30 letters (65.2\%). Absent or unclear instruction to the clinics could lead to lack of accountability and responsibility when it comes to the clinical management of the patient. The patient could quite simply be lost to follow-up because the clinic could assume that the patient is still under the management and care of the hospital or vice versa. Van Walraven ${ }^{6}$ argues that delayed or inappropriate communication between the hospital-based physicians and the primary care physicians at the point of hospital discharge may negatively affect the continuity of care and contribute to adverse events.

Brez $^{19}$ identified the utilisation of an individualised plan of care for diabetic patients and a structured discharge letter as tools to achieve improved communication between hospital-based doctors and primary care practitioners. As suggested by Brez, the plan of care would support transitional care if it included the most recent laboratory results, information on local resources and support services, current treatment regimens and targets and specific advice for follow-up and next treatment steps. ${ }^{19}$ Brez's study supports the need to have information regarding the clinical management of patients and follow-up as a means to ensure the continuity of care. ${ }^{19}$

The feedback letters in this study lacked information on the overall presenting clinical picture and the condition of the patient and as a result fail to include the reasons why the patient was treated at the hospital. The information relating to the clinical management of the patient is also very poor. The primary reason why the patient was sent to the hospital is that the patient could not be managed at a clinic level in the first place and therefore further tests and more specialised forms of treatment or management were required. The clinics need the hospitals to provide care that cannot be given at the clinic level, yet the hospital does not give information regarding 
what has been done at their facility. It is also evident there was missing information in some letters as to whether the clinic or the hospital was supposed to follow-up on the patient and, if it was the clinic, there was not clear instruction on what were they supposed to do in terms of follow-up, e.g. monitor blood pressure, patient health education, continue treatment as prescribed etc.

Treatment given to patients is also important to record because it implies that there were specialised interventions done by the hospital. Feedback letters that contain this information are a rich source of learning opportunities for the clinicians or nurse practitioners at the PHC clinics. Gagliardi ${ }^{20}$ reported that GPs were receptive to the use of the feedback letters as a source of learning but the feedback letters from specialists to GPs had very little educational content. This lost opportunity could result in unnecessary referrals of other patients with similar clinical presentations - a key consideration to prevent upward pressure on the system.

From the patients' perspective, the largely uneducated population in Metsweding cannot be expected to convey instructions to the referring PHC clinics. The majority of patients come from a poor background and rely on public transport. Reducing the travel costs for patients by avoiding unnecessary follow-up visits to the hospital can be kept to a minimum by giving the patient a letter with clear instructions for the clinic.

A limitation of the study is that the study excluded patient records of those who were referred by general practitioners in private practice and also the records of patients who take their records home. A second limitation is that the study did not compare the adequacy of the feedback letters in terms of variables such as age, gender and type of referral, e.g. emergency or elective. The final sample of eligible patient records was small and therefore limits generalisability of the findings. The adequacy of the feedback in terms of time taken for feedback to reach the clinics could not be determined as the patients deliver the letters themselves. Abstraction errors are possible as only one person abstracted the variables from the data. A final limitation is that the study did not establish whether there was a difference between the different district hospitals in terms of feedback.

\section{Conclusion}

The feedback rate in Metsweding from first referral hospitals to PHC clinics is scarcely higher than studies from other countries and reflects poorly on the referral system. The feedback letters that were examined were incomplete and often did not contain the standard and crucial elements of feedback letters described in the international literature. Opportunities for primary care practitioners to learn are lost. The lack of continuum of critical information about patients who are referred to hospitals is ineffective in improving the quality of care of the patient and could lead to wastage of resources. Patients bear the consequences as they depend on the system greatly and the breakdown of communication within the system can result in unnecessary clinic and hospital visits that can stretch patients' overstretched socio-economic capacity. The results in this study are no better when compared with results from studies in other low-resource countries. A simple standard feedback template that contains all the details that are considered important by both the PHC clinics and hospitals could go a long way to fill in the blanks.

Acknowledgements - The authors would like to thank Dr Nicholas Crisp who reviewed the article as part of a mini-dissertation and Prof Samuel Manda for assistance in calculating the sample size. They would also like to acknowledge the clinic clerks at the Metsweding district clinics who helped to sort the files that met the criteria for the study for their kind assistance.
Authors' contributions - TLL (University of Pretoria) was the project leader and conducted all aspects of the research as part of her master's studies; JEW (University of Pretoria) provided intellectual input in the conception and design of the study and revised the article.

Conflict of interest - The authors declare that they have no financial or personal relationship(s) that may have inappropriately influenced them in writing this paper.

\section{References}

1. WHO Study Group on the Functions of Hospitals at the First Referral Level, World Health Organization. The hospital in rural and urban districts: report of a WHO Study Group on the functions of hospitals at the first referral level. Geneva: World Health Organization; 1992.

2. World Health Organization. Managerial process for national health development: guiding principles for use in support of strategies for health for all by the year 2000. Geneva: World Health Organization; 1981.

3. Abate B, Enqueselassie F. Information use in patients referred at Tikur Anbessa specialized hospital, Addis Ababa. Ethiopia. Ethiop Med J. 2010 Apr;48(2):123-35.

4. Abdelwahid HA, Al-Shahrani SI, Elsaba MS. Patterns of referral in the family medicine department in southeastern Saudi Arabia. Saudi Med J. 2010 Aug;30(8):923-30.

5. Westerman RF, Hull FM, Bezenes PD, et al. A study of communication between general practitioners and specialists. Br J Gen Pract. 1990 Nov [cited 2013 Feb 02];40(340):445-9.

6. Walraven C, Mamdani M, Fang J, et al. Continuity of care and patient outcomes after hospital discharge. J Gen Intern Med. 2004 June [cited 2012 Mar 3];19(6):624-31. http://dx.doi.org/10.1111/j.15251497.2004.30082.x

7. Kattan M, Crain EF, Steinbach S, et al. Quality of inpatient care for asthma: challenges and opportunities. Pediatrics. 2008 Dec 6;122(6):136970. http://dx.doi.org/10.1542/peds.2008-2787

8. Bossyns $\mathrm{P}, \mathrm{Abache} \mathrm{R}, \mathrm{Abdoulage} \mathrm{M}$, et al. Monitoring the referral system through benchmarking in rural Niger: an evaluation of the functional relation between health centres and the district hospital. BMC. 2006 April 12;51(6):879-87.

9. Siddiqi S, Kielmann A, Khan $M$, et al. The effectiveness of patient referral in Pakistan. Health Policy Plan. 2001;16(2):193-8. Available from: http://heapol.oxfordjournals.org/content/16/2/193.short.10.1093/ heapol/16.2.193

10. Ohara K, Melendez V, Uehara N, et al. Study of a patient referral system in the Republic of Honduras. Health Policy Plan. JICA. 1992;13(4):433-5.

11. Fletcher CM. Communication in medicine. London: Nuffield Provincial Hospital Trust; 1973.

12. Jarallah JS. Referral from primary care to hospitals in Saudi Arabia: quality of referral letters and feedback reports. J Family Community Med. 1998 Jul [cited 2013 June 5];5(2):15-22.

13. Young DW, Parker J, Davis WA, et al. Out-patient letters: requirements and contents. Effective Health Care. 1998;2:225-8.

14. Hart JT, Marinker M. An exchange of letters. London: MSD Foundation; 1985.

15. Newton J, Eccles M, Hutchinson A. Communication between general practitioners and consultants: what should their letters contain? BMJ. 1992 Mar [cited 2012 Aug 7];304(6830):821-4. http://dx.doi. org/10.1136/bmj.304.6830.821

16. Mojaki ME, Basu D, Letskokgohka ME, et al. Referral steps in district health system are side-stepped. SAMJ. 2011 Feb;101(2):109.

17. Loubster J, Maree G, Kalele P. Metsweding status quo report. Report 1 of the Metsweding management framework and environment plan. 2011:1-119.

18. vanWalraven C, Anthony L, Weinberg MB. Quality assessment of a discharge summary system. CMAJ. 2005 May 1 [cited 2013 Apr 2];152(9):1437-42.

19. Brez S, Rowan M, Malcom J, et al. Transition from specialist to primary diabetes care: a qualitative study of perspectives of primary care physicians. BMC Fam Pract. 2009 [cited May 1];39(10):1471-2296.

20. Gagliardi A. Use of referral reply letters for continuing medical education: A review. J Contin Educ Health Prof. 2002;22(4):222-9. http://dx.doi.org/10.1002/(ISSN)1554-558X 